\title{
APLICABILIDADE DE INOVAÇÕES E TECNOLOGIAS ASSISTENCIAIS PARA A SEGURANÇA DO PACIENTE: REVISÃO INTEGRATIVA
}

\author{
Virna Ribeiro Feitosa Cestari ${ }^{1}$, Marília Araripe Ferreira ${ }^{2}$, Thiago Santos Garces ${ }^{3}$, Thereza Maria Magalhães \\ Moreira $^{4}$, Vera Lúcia Mendes de Paula Pessoa ${ }^{5}$, Islene Victor Barbosa ${ }^{6}$
}

\begin{abstract}
RESUMO: Objetivou-se identificar as tecnologias utilizadas pelo enfermeiro para promoção da segurança do paciente no contexto hospitalar. Revisão integrativa, com busca de artigos em bases de dados na área da saúde, no período de janeiro a fevereiro de 2016. Foram selecionados 20 artigos, divididos segundo as tecnologias assistenciais utilizadas. No intuito de promover a segurança do paciente, os enfermeiros utilizam tecnologias duras - implantação de registros eletrônicos, bombas de infusão, sensores e sistemas computadorizados e de alerta; incorporam as boas práticas em saúde - construção de instrumentos voltados para segurança do paciente e identificação de riscos; e realizam/participam de cursos de capacitação profissional - cálculo e administração de medicamentos e infusões intravenosas e higienização das mãos. Conclui-se que o enfermeiro é um profissional que tem se preocupado com a segurança do paciente, o que reforça a necessidade da realização de mais estudos que tenham como foco a utilização de estratégias que promovam tal prática.

DESCRITORES: Segurança do paciente; Enfermagem; Tecnologia biomédica; Assistência à saúde; Capacitação em serviço.

\section{APPLICABILITY OF ASSISTIVE INNOVATIONS AND TECHNOLOGIES FOR PATIENT SAFETY: INTEGRATIVE REVIEW}

ABSTRACT: The present study aimed to identify the technologies used by nurses to promote patient safety in hospital settings. Integrative review, with search of articles in databases in the health area, from January to February, 2016. Twenty articles were selected and sorted according to the assistive technologies used. In the promotion of patient safety, nurses use hard technologies implementation of electronic records, automated drug infusion pumps, sensors and computer-based monitoring systems. They also incorporate good practices in health - construction of instruments targeted to patient safety and identification of risks, and promote/ participate in professional training courses - calculation and administration of medications and intravenous infusions and hand hygiene. It is concluded that nursing professionals are concerned with patient safety, which reinforces the need to conduct further studies on the use of relevant strategies.

DESCRIPTORS: Patient safety; Nursing; Biomedical technology; Health care; In-service training.

\section{APLICABILIDAD DE INOVACIONES Y TECNOLOGÍAS DE ASSTENCIA PARA SEGURIDAD DEL PACIENTE: REVISIÓN INTEGRATIVA}

RESUMEN: Estudio cuyo propósito fue identificar las tecnologías utilizadas por el enfermero para promover la seguridad del paciente en el contexto hospitalar. Revisión integrativa, con búsqueda de artículos en bases de datos en el área de la salud, en periodo de enero a febrero de 2016. Se seleccionaron 20 artículos, organizados de acuerdo a las tecnologías asistenciales utilizadas. Para promover la seguridad del paciente, los enfermeros utilizan tecnologías duras - implantación de registros electrónicos, bombas de infusión, sensores y sistemas computarizados y de alerta; añaden las buenas prácticas en salud - construcción de instrumentos para dar seguridad al paciente, así como para identificación de riesgos; y realizan/participan de cursos de capacitación profesional - cálculo y administración de medicamentos e infusiones intravenosas, además de higienización de las manos. Se concluye que el enfermero es un profesional que se preocupa con la seguridad del paciente, lo que resalta la necesidad de realización de más estudios cuya énfasis sea el uso de estrategias que promovan esa práctica.

DESCRIPTORES: Seguridad del paciente; Enfermería; Tecnología biomédica; Asistencia a la salud; Capacitación en servicio.

${ }^{1}$ Enfermeira. Mestranda em Cuidados Clínicos em Enfermagem e Saúde. Universidade Estadual do Ceará. Fortaleza, CE, Brasil. ${ }^{2}$ Enfermeira. Residente em Assistência em Diabetes. Hospital Universitário Walter Cantídio, Universidade Federal do Ceará. Fortaleza, CE, Brasil.

${ }^{3}$ Enfermeiro. Mestrando em Cuidados Clínicos em Enfermagem e Saúde. Universidade Estadual do Ceará. Fortaleza, CE, Brasil. ${ }^{4}$ Enfermeira. Pós-doutora em Enfermagem. Docente da Universidade Estadual do Ceará. Fortaleza, CE, Brasil.

${ }^{5}$ Enfermeira. Doutora em Enfermagem. Docente da Universidade Estadual do Ceará. Fortaleza, CE, Brasil.

${ }^{6}$ Enfermeira. Doutora em Enfermagem. Docente da Universidade de Fortaleza. Fortaleza, CE, Brasil.

Autor Correspondente:

Virna Ribeiro Feitosa Cestari

Universidade Estadual do Ceará

Av. Dr. Silas Munguba, 1700 - 60741-000 - Fortaleza, CE, Brasil

E-mail: virnarfcestari@gmail.com
Recebido: 25/02/2016

Finalizado: 30/06/2017 


\section{- INTRODUÇÃO}

A segurança do paciente pode ser definida como o ato de evitar, prevenir e melhorar os resultados adversos ou as lesões associadas aos cuidados em saúde, usando métodos baseados em evidências. É um dos assuntos prioritários na área da saúde mundialmente e vem recebendo crescente atenção, pois, como o processo do cuidado à saúde tem se tornado cada vez mais complexo, eleva o potencial para ocorrência de acidentes, erros e/ou eventos adversos $(E A)^{(1)}$.

Dados da literatura revelam que milhões de pessoas no mundo sofrem lesões desabilitantes e mortes decorrentes de práticas em saúde inseguras. Tal estimativa advém de estudos conduzidos em países desenvolvidos, com sistemas de saúde mais estruturados do que os de países em desenvolvimento, nos quais dados epidemiológicos ainda são escassos. Em 1999 a temática ganhou destaque a partir de dados publicados pelo Instituto de Pesquisa dos Estados Unidos, que revelou que os erros de cuidado à saúde causavam de 44 a 98 mil eventos adversos, anualmente, em hospitais daquele país. Na Europa, estimou-se que $10,8 \%$ dos pacientes hospitalizados foram acometidos por eventos adversos, dos quais $46 \%$ poderiam ter sido prevenidos ${ }^{(2)}$.

As consequências oriundas da ruptura da segurança do paciente aumentam os gastos com a saúde, o tempo de internação, podem implicar em complicações e óbitos, o que ocasiona a redução da confiança do usuário no sistema de saúde, acarreta danos psicológicos e, muitas vezes, encargos jurídicos ${ }^{(3)}$.

O enfermeiro tem papel fundamental na promoção da segurança do paciente durante o processo assistencial, visto ser este o profissional responsável por coordenar a assistência de enfermagem prestada. Ademais, é crescente o número de enfermeiros atuantes nas instituições e a sua responsabilidade nos cuidados aos pacientes durante as 24 horas ${ }^{(4)}$. Nessa perspectiva, a prestação de assistência ao paciente tem exigido da enfermagem atuação extremamente complexa, evocando a necessidade da mesma se capacitar cientificamente e se comprometer eticamente com ações sistêmicas de avaliação e prevenção, e tentando viabilizar a redução de desfechos indesejados, assim como a garantia da qualidade do cuidado prestado ${ }^{(5)}$.

Considerando a magnitude do tema, destaca-se a necessidade da produção tecnológica de inovações na área da saúde e enfermagem. Quando usada a favor da saúde, a inovação tecnológica contribui diretamente com a qualidade, efetividade e segurança do cuidado, resultando em um viver saudável ${ }^{(4)}$. Por conseguinte, surgiu a necessidade de se investigar quais as inovações tecnológicas têm sido utilizadas pelo enfermeiro na busca pela segurança do paciente.

Com o intuito de contribuir com pesquisas que deem visibilidade à problemática, o presente estudo teve como objetivo identificar as tecnologias utilizadas pelo enfermeiro para promoção da segurança do paciente no contexto hospitalar.

\section{MÉTODO}

Revisão integrativa da literatura, cuja análise dos estudos selecionados foi norteada pela pergunta de pesquisa: quais as tecnologias utilizadas pelo enfermeiro para a promoção da segurança do paciente?

Optou-se pelas bases de dados: Literatura Latino-Americana e do Caribe em Ciências da Saúde (LILACS), Base de Dados de Enfermagem (BDENF), Índice Bibliográfico Espanhol de Ciências da Saúde (IBECS), Scientific Eletronic Library Online (SciELO), Medical Literature Analysis and Retrieval System on-line (MEDLINE) e National Library of Medicine (PubMed), utilizando os descritores patient safety, technology e nursing, de acordo com a terminologia MeSH. A equação de busca foi "patient safety" $A N D$ "nursing" OR "patient safety" AND "technology" AND "nursing".

Os critérios de inclusão delimitados para pré-seleção dos estudos foram: artigos sobre a temática segurança do paciente; produzidos por enfermeiros; publicados em periódicos nacionais e internacionais nos últimos seis anos (2011-2016); que contemplassem os objetivos propostos; em língua inglesa, portuguesa, espanhola e francesa; e disponíveis eletronicamente na íntegra. Foram excluídos capítulos de livros, teses de doutorado, dissertações de mestrado, editoriais, cartas ao editor 
e artigos de reflexão, revisão e atualização.

A partir das buscas nas bases de dados e aplicação dos critérios de elegibilidade, foram incluídos 20 estudos na presente revisão. A Figura 1 apresenta o fluxograma de seleção desses estudos.

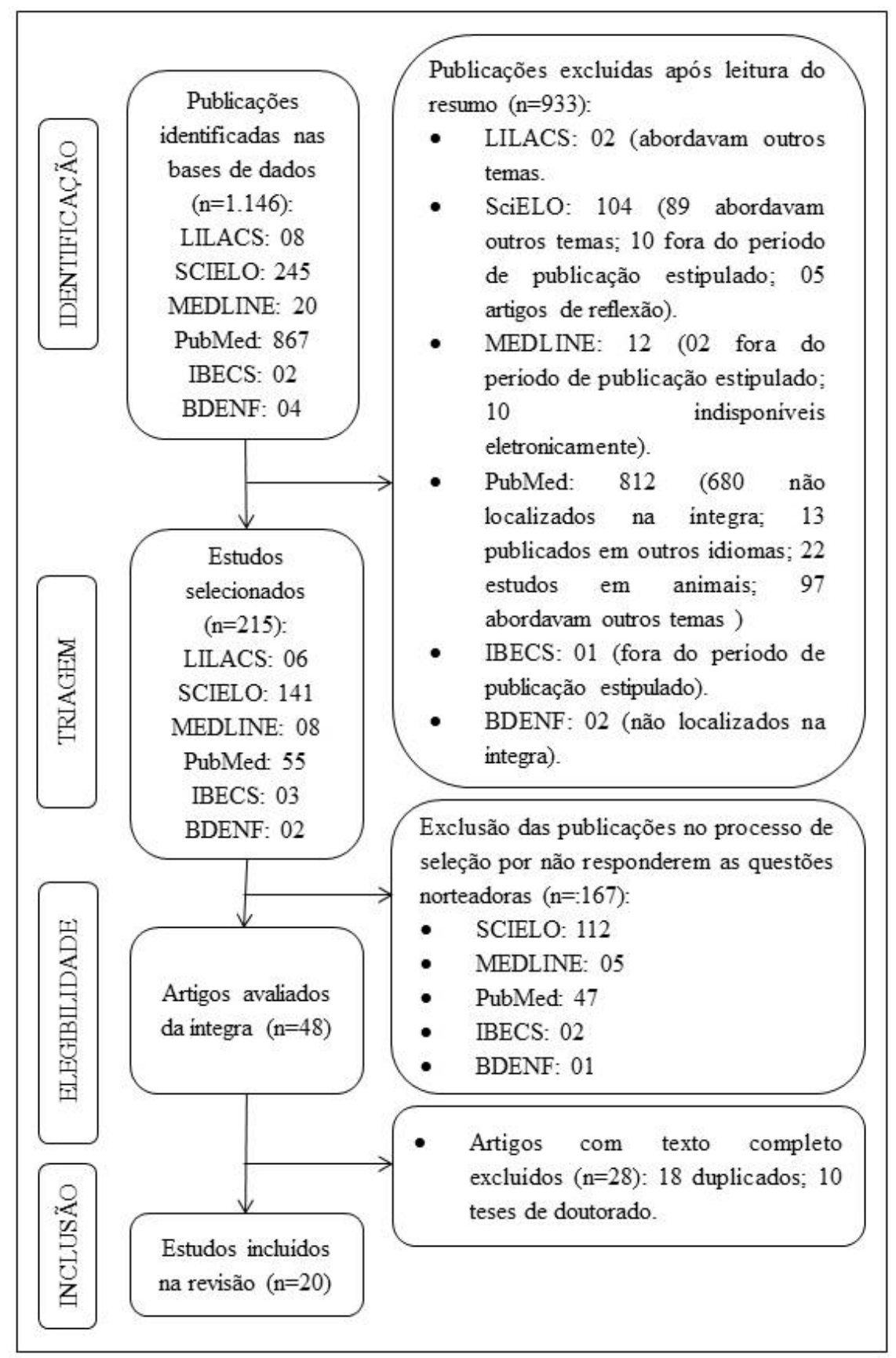

Figura 1 - Fluxograma da seleção dos estudos segundo o PRISMA. Fortaleza, CE, Brasil, 2016

Inicialmente, o processo de seleção dos estudos foi executado por meio da leitura minuciosa de títulos e resumos, de modo que foram para seleção final os estudos que atendiam aos critérios de inclusão supracitados. Para seleção final dos artigos, foi realizada análise crítica e detalhada por dois revisores e um terceiro foi consultado em caso de discordância na seleção das pesquisas.

Dos estudos selecionados para a amostra final, foram coletados os seguintes dados: país, periódico e ano de publicação, delineamento metodológico, nível de evidência (NE), população-alvo (adulta, pediátrica ou profissionais de enfermagem), extensão do estudo (unicêntrico ou multicêntrico) e tema do estudo. Os dados foram inseridos no programa da Microsoft Exce ${ }^{\circledast}{ }^{2010}$, submetidos à análise descritiva e a síntese dos resultados foi apresentada em quadros. 
Foi possível observar maior quantitativo de publicações no ano de 2014, com dez artigos (50\%). Os estudos desenvolvidos e publicados no Brasil totalizaram 12 (60\%) dos artigos, sendo, dessa forma, o português o idioma mais frequente de publicação (Quadro 1). A região Sudeste foi a que apresentou maior número de pesquisas envolvendo a temática em estudo (cinco; 25\%); seguidas da Sul (quatro; $20 \%$ ); Nordeste (dois; $10 \%$ ) e Centro-Oeste (um; $5 \%$ ).

Quadro 1 - Caracterização dos estudos selecionados. Fortaleza, CE, Brasil, 2016

\begin{tabular}{|c|c|c|c|c|c|}
\hline Estudo & País & Periódico/ano de publicação & $\begin{array}{l}\text { Delineamento } \\
\text { metodológico }\end{array}$ & NE & $\begin{array}{c}\text { Extensão do } \\
\text { estudo }\end{array}$ \\
\hline $\mathrm{A} 1^{(6)}$ & Estados Unidos & Journal of Nurse Administration/2011 & Transversal & III & Multicêntrico \\
\hline $\mathrm{A} 2^{(7)}$ & Brasil & $\begin{array}{l}\text { Revista Latino-Americana de } \\
\text { Enfermagem } / 2012\end{array}$ & Descritivo & $\mathrm{V}$ & Unicêntrico \\
\hline $\mathrm{A} 3^{(8)}$ & Canadá & $\begin{array}{l}\text { Ontario Health Technology Assessment } \\
\text { Series/2012 }\end{array}$ & Transversal & III & Multicêntrico \\
\hline $\mathrm{A} 4^{(9)}$ & Estados Unidos & Nursing for Women's Health/2012 & Transversal & III & Multicêntrico \\
\hline $\mathrm{A} 5^{(10)}$ & Brasil & Cadernos de Saúde Pública/2012 & Metodológico & $\mathrm{V}$ & Unicêntrico \\
\hline $\mathrm{A} 6^{111}$ & Japão & Methods of Information in Medicine/2013 & Transversal & $\mathrm{V}$ & Unicêntrico \\
\hline $\mathrm{A} 7^{(12)}$ & Brasil & $\begin{array}{l}\text { Revista Latino-Americana de } \\
\text { Enfermagem/2013 }\end{array}$ & Metodológico & $\mathrm{V}$ & Unicêntrico \\
\hline $\mathrm{A} 8^{(13)}$ & Brasil & Revista Gaúcha de Enfermagem/2013 & Estudo analítico & III & Unicêntrico \\
\hline $\mathrm{A}^{(14)}$ & Brasil & $\begin{array}{l}\text { Revista Latino-Americana de } \\
\text { Enfermagem/2013 }\end{array}$ & Metodológico & $\mathrm{V}$ & Unicêntrico \\
\hline $\mathrm{A} 10^{(15)}$ & Brasil & Escola Anna Nery/2014 & Descritivo & $\mathrm{V}$ & Unicêntrico \\
\hline $\mathrm{A} 11^{(16)}$ & Nigéria & $\begin{array}{l}\text { The Brazilian Journal of Infectious } \\
\text { Diseases/2014 }\end{array}$ & Transversal & III & Unicêntrico \\
\hline $\mathrm{A} 12^{(17)}$ & Noruega & BMJ Open/2014 & $\begin{array}{l}\text { Ensaio clínico } \\
\text { randomizado }\end{array}$ & $\mathrm{I}$ & Unicêntrico \\
\hline $\mathrm{A} 13^{(18)}$ & Brasil & Revista Escola de Enfermagem da USP/2014 & Transversal & III & Unicêntrico \\
\hline A14 ${ }^{(19)}$ & Brasil & Acta Paulista de Enfermagem/2014 & Transversal & III & Unicêntrico \\
\hline $\mathrm{A} 15^{(20)}$ & Brasil & Revista Escola de Enfermagem da USP/2014 & $\begin{array}{l}\text { Híbrido: produção } \\
\text { tecnológica } \\
\text { e pesquisa } \\
\text { metodológica }\end{array}$ & $\mathrm{V}$ & Unicêntrico \\
\hline $\mathrm{A} 16^{(21)}$ & Brasil & Acta Paulista de Enfermagem/2014 & Longitudinal & IV & Multicêntrico \\
\hline $\mathrm{A} 17^{(22)}$ & EUA & Journal for Healthcare Quality/2014 & Transversal & III & Unicêntrico \\
\hline $\mathrm{A} 18^{(23)}$ & Arábia Saudita & $\begin{array}{l}\text { International Journal of Electronic } \\
\text { Healthcare/2014 }\end{array}$ & Transversal & III & Unicêntrico \\
\hline A19 ${ }^{(24)}$ & Minas Gerais & Texto e Contexto Enfermagem/2015 & Descritivo & $\mathrm{V}$ & Unicêntrico \\
\hline $\mathrm{A} 20^{(25)}$ & Brasil & Revista Escola de Enfermagem da USP/2015 & Descritivo & $\mathrm{V}$ & Unicêntrico \\
\hline
\end{tabular}

Houve predomínio de estudos transversais, nove (45\%); de abordagem quantitativa, 17 (85\%); unicêntricos, 16 (80\%); e 13 (65\%) estudos tinham os adultos como principal população-alvo. Para o nível de evidência, foi utilizada a classificação a partir do delineamento experimental ${ }^{(26)}$, constatando equivalência entre o quantitativo de estudos publicados com NE III (evidências obtidas de estudos sem randomização controlados, coorte ou de seguimento) e $\mathrm{V}$ (evidências originárias de estudos descritivos), com nove (45\%) cada.

No que tange às temáticas, $12(60 \%)$ tratavam da incorporação das boas práticas em saúde para 
a promoção da segurança do paciente; cinco (20\%), do uso de tecnologias duras; e três (15\%), da capacitação do profissional enfermeiro (Quadro 2).

Quadro 2 - Distribuição dos estudos segundo temas abordados. Fortaleza, CE, Brasil, 2016

\begin{tabular}{|l|l|}
\hline Tema do estudo & Estratégias utilizadas pelos enfermeiros \\
\hline Uso de tecnologias duras & $\begin{array}{l}\text { Implantação de registros eletrônicos de saúde (A1); } \\
\text { Bombas de infusão inteligentes (A4); } \\
\text { Sistemas de sensores de coleta de sinais vitais (A7); } \\
\end{array}$ \\
$\begin{array}{l}\text { Sistemas de alerta (A15); } \\
\text { Sistemas computadorizados de entrada do paciente (A18). }\end{array}$ \\
\hline $\begin{array}{l}\text { Incorporação das boas práticas } \\
\text { em saúde para a promoção da } \\
\text { segurança do paciente }\end{array}$ & $\begin{array}{l}\text { Construção/validação/adaptação de instrumentos voltados para a segurança } \\
\text { do paciente (A2, A5, A7, A8, A9, A16, A19); } \\
\text { Identificação dos riscos para a segurança (A10); } \\
\text { Identificação de medicamentos (A14, A17, A20). }\end{array}$ \\
\hline Capacitação do enfermeiro & $\begin{array}{l}\text { Administração de infusões intravenosas (A3); } \\
\text { Promoção da higienização das mãos (A11); } \\
\text { Cálculo de medicamentos (A12). }\end{array}$ \\
\hline
\end{tabular}

\section{DISCUSSÃO}

\section{Uso de tecnologias duras}

Dentre as tecnologias do cuidado, as duras ou trabalho morto referem-se ao instrumental complexo em seu conjunto, englobando todos os equipamentos para tratamentos, exames e a organização das informações ${ }^{(11,23)}$.

A implantação dos registros de saúde eletrônicos é ferramenta para qualificar os cuidados prestados e a efetivar segurança dos doentes. Oferece nível de detalhamento para relatórios de transferência, avaliação de administradores, permite a economia de gastos referentes à redução de erros nas medicações, tempo gasto na documentação, melhoria dos registros de enfermagem e na comunicação com a equipe ${ }^{(6)}$. Um estudo ${ }^{(6)}$ realizado em 316 hospitais dos EUA evidenciou que apenas $21(7 \%)$ tinham um sistema de registros eletrônicos implementados em todas as unidades de atendimento ao paciente, e os enfermeiros desses hospitais foram menos propensos a relatar desfechos desfavoráveis quando comparados aos que não utilizavam essa tecnologia.

As tecnologias duras também têm sido amplamente utilizadas no processo de medicação. Os erros na administração dos medicamentos são muito frequentes nas unidades de saúde, principalmente quando está relacionado a dosagens precisas e medicações nocivas ao corpo humano. Com o objetivo de amenizar os erros e aplicar uma dose mais fidedigna, foram criadas as bombas inteligentes para identificar as características especificas dos pacientes, sugerir informações sobre a diluição, taxas de infusão recomendadas, cálculos de dosagem de acordo com o peso, verificação prévia de fatores imprescindíveis para assegurar a administração do medicamento, evitando assim uma situação insegura. A nova tecnologia evidenciou uma redução de erros de 3,1 para 0,8 por 1.000 doses $^{(9)}$.

Outra tecnologia implantada no intuito de promover a segurança do paciente é o Processo de Enfermagem Informatizado (PEI), embasado na Classificação Internacional para as Práticas de Enfermagem (CIPE) para UTI. A proposta buscou a recomendação de Diagnósticos de Enfermagem (DE), identificação das alterações clínicas possíveis e intervenções adequadas para cada caso, por meio de mensagens de alerta para segurança ${ }^{(20)}$. Os autores constataram a eficácia desse programa e sugeriram a adição de mais alertas potenciais para: pneumonia iatrogênica, infecções secundárias ao cuidado prestado, deiscência de sutura no pós-operatório de pacientes com cirurgia abdominal ou pélvica, perda de acesso vascular e extubação endotraqueal. 
Percebe-se portanto que, com a formulação de sinais de alerta, com suas devidas orientações, o profissional permanece preparado para situações que comprometam a segurança do paciente, tendo como suporte um processo que orienta suas ações, a fim de garantir uma prática baseada em evidências científicas, minimizando e prevenindo os EA.

\section{Incorporação das boas práticas em saúde}

As boas práticas em saúde envolvem as ações adotadas pelas instituições de saúde bem como seus funcionários, no intuito de possibilitar a identificação e resolução de problemas com segurança e agilidade, de forma a propiciar melhor assistência ${ }^{(21)}$.

Os EA são resultados de diversos fatores desfavoráveis, como a estrutura física do local de trabalho; indisposição de recursos materiais; jornadas de trabalho abusivas; excesso de pacientes por enfermeiro; ausência de educação continuada; falta de estímulos à notificação de EA, entre outros ${ }^{(14,25)}$. Justificase, portanto, a construção e validação de diversas escalas de predisposição para ocorrência de EA, buscando a melhoria do cuidado(7) .

Envolver o paciente e família na sua própria segurança é uma estratégia de prevenção de EA, principalmente quando se trata de crianças. Pensando nisso, um estudo metodológico ${ }^{(12)}$ teve como objetivo elaborar e validar um checklist com intervenções pré-operatórias relacionadas à segurança do paciente, a ser preenchido pela criança e família. O instrumento demonstrou lacunas na comunicação entre profissional, crianças e familiares, como a utilização de terminologias técnicas, deficiência de registros de dados no prontuário, atitudes do profissional frente aos questionamentos da criança ou familiar, e informações deficientes. Portanto, são necessárias melhorias nas orientações prestadas no pré-operatório e adaptações da linguagem utilizada.

A implantação do checklist é de baixo custo e demora cerca de três minutos para sua aplicação e a dificuldade em sua aplicação está localizada na equipe cirúrgica(10). O processo conta com um responsável pela aplicação, sendo o enfermeiro o indicado para orientar a checagem; o profissional deve ter autoridade plena sobre o processo cirúrgico, estando apto a interromper o procedimento ou impedir o avanço, se julgar insatisfatório algum dos itens ${ }^{(13)}$.

A construção e implementação de roteiros também se mostraram eficazes para assegurar a segurança do paciente. Estudo ${ }^{(24)}$ realizado em Minas Gerais objetivou elaborar roteiro para identificar os principais incidentes no transporte intra-hospitalar de pacientes internados na UTI. Os autores verificaram que os incidentes estavam relacionados a panes e problemas de mau funcionamento nos equipamentos e dispositivos, sendo as circunstâncias de risco frequentes: variação da pressão arterial, agitação, queda da saturação arterial periférica e taquicardia.

Outro estudo ${ }^{(21)}$ desenvolvido em três unidades de terapia intensiva (UTI) de São Paulo objetivou analisar as boas práticas em saúde. Ao serem comparadas, as unidades apresentaram diferenças importantes na mudança de decúbito; prevenções de quedas; prevenção de infecção hospitalar, entre outros. Os itens que apresentaram medidas eficazes em mais de $90 \%$ nas três instituições foram: colchão caixa de ovo; paciente sentado e grades elevadas para prevenir quedas; identificação do leito; cabeceira elevada acima de $30^{\circ}$; torneirinhas protegidas com "cone luer" e bolsa coletora de diurese abaixo do nível da bexiga. Diante disso, as instituições devem manter e aprimorar uma política de gerenciamento de riscos, prevenindo erros e buscando a melhoria da assistência.

Pesquisa ${ }^{(15)}$ realizada no Ceará revelou que a maior preocupação dos enfermeiros era em relação ao risco de quedas, transmissão de infecção e EA relacionados ao procedimento cirúrgico. Os enfermeiros são os principais responsáveis pela implementação das práticas seguras nos serviços, indicando a qualidade do cuidado prestado. Comprovando tal fato, esses profissionais demonstraram estar atentos às metas internacionais, bem como aos protocolos de cirurgia segura, realizando os checklists e a incorporação das evidências científicas.

Além das estratégias específicas voltadas para a segurança, o enfermeiro pode implementá-la no Processo de Enfermagem (PE), método de trabalho sistematizado e embasado cientificamente que orienta a assistência e a documentação, facilitando a avaliação do indivíduo. A fim de padronizar o 
PE, são usados instrumentos como os Diagnósticos de Enfermagem (DE) NANDA Internacional, o qual nomeia o problema vivenciado, e o Nursing Interventions Classification (NIC) que apresenta as intervenções prioritárias para este $\mathrm{DE}^{(18)}$.

Quanto à administração de medicamentos, a legibilidade das prescrições deve ser prezada pelo profissional prescritor com todas as informações sugeridas, assinatura e conselho profissional, bem como o diálogo deve ocorrer nesse momento, deixando claro para equipe as alterações e suspensões, evitando assim falha de comunicação ${ }^{(19,25)}$.

\section{Capacitação do enfermeiro}

A lavagem das mãos (LM) é uma estratégia simples para a segurança do paciente, evitando infecções para o doente e o profissional. Um estudo ${ }^{(16)}$ desenvolvido na Nigéria teve como objetivo treinar 202 trabalhadores em saúde para a higiene correta das mãos. Após o treinamento, os autores constaram adesão de $75,3 \%$ na LM, principalmente, após o contato com fluidos corporais (75,3\%) e após tocar no paciente $(73,6 \%)$. A taxa de adesão foi maior em enfermeiros $(72,9 \%)$ quando comparados aos médicos $(59,7 \%)$ e parteiras $(65 \%)$.

Outra preocupação evidenciada nos estudos selecionados foram os erros nos cálculos das doses de medicamentos. Um estudo ${ }^{(17)}$ randomizado realizado na Noruega com 183 participantes revelou que a metade dos médicos é incapaz de realizar cálculo de medicamentos e que, para se tornar um enfermeiro, são necessários testes sem falhas dessa prática. A implementação de curso de cálculo de medicamentos levou à melhoria desta prática, 37 (20,2\%) dos participantes obtiveram nota excelente.

A capacitação mostrou-se eficaz também em um estudo ${ }^{(8)}$ desenvolvido em Ontario, que buscou identificar as dificuldades dos enfermeiros no manuseio do paciente com acesso intravenoso. Depois de identificadas, foram realizadas intervenções educativas por meio de telefonemas.

Os profissionais de saúde necessitam de suporte da educação continuada, buscando a atualização nas novas diretrizes, como manusear equipamentos modernos, formas de administrar medicamentos recentes, estratégias para melhorar a assistência e como trabalhar em equipe.

\section{CONSIDERAÇÕES FINAIS}

Constatou-se que a enfermagem é profissão de destaque no que se refere à segurança do paciente nos mais variados cuidados. Essa atribuição representa grande desafio, pois exige trabalho em conjunto com a equipe multiprofissional. Espera-se do enfermeiro que sua tomada de decisão vise sempre à segurança do paciente e que possua a prática embasada na pesquisa e em evidências.

Dentre as contribuições do enfermeiro para a segurança do paciente, destacaram-se os cuidados de enfermagem como a higienização das mãos, cuidados no manejo e administração de medicamentos, identificação de riscos à segurança do paciente, uso de sistemas de informação e aplicabilidade de instrumentos e escalas para avaliar a segurança dos pacientes.

\section{REFERÊNCIAS}

1. de Souza VS, Kawamoto AM, de Oliveira JLC, Tonini NS, Fernandes LM, Nicola AL. Erros e eventos adversos: a interface com a cultura de segurança dos profissionais de saúde. Cogitare Enferm. [Internet] 2015;20(3) [acesso em 10 fev 2016]. Disponível: http://dx.doi.org/10.5380/ce.v20i3.40687.

2. François $P$, Sellier E, Imburchia F, Mallaret MR. Le comité de retour d'expérience (CREX): une méthod pour I'améliorantion de la sécurité des soins. Rev Epidemiol Sante Publique. [Internet] 2013;61(2)[acesso em 10 fev 2016]. https://doi.org/10.1016/j.respe.2012.09.001.

3. Nunes FDO, Barros LAA, Azevedo RM, Paiva SS. Segurança do paciente: como a enfermagem vem contribuindo para a questão? J. res.: fundam. care. online. [Internet] 2014;6(2) [acesso em 14 fev 2016]. Disponível:http://dx.doi. 
4. Caldana G, Guirardello EB, Urbanetto JS, Peterlini MAS, Gabriel CS. Rede brasileira de enfermagem e segurança do paciente: desafios e perspectivas. Texto Contexto Enferm. [Internet] 2015;24(3) [acesso em 10 fev 2016]. Disponível: http://dx.doi.org/10.1590/0104-070720150001980014.

5. Bogarin DF, Zanetti ACB, Brito MFP, Machado JP, Gabriel CS, Bernardes A. Segurança do paciente: conhecimento de alunos de graduação em enfermagem. Cogitare Enferm. [Internet] 2014;19(3) [acesso em 10 fev 2016]. Disponível: http://dx.doi.org/10.5380/ce.v19i3.33308.

6. Kutney-Lee A, Kelly D. The effect of hospital electronic health record adoption on nurse-assessed quality of care and patient safety. J Nurs Adm. [Internet] 2011;41(11) [acesso em 12 fev 2016]. Disponível: http://dx.doi. org/10.1097/NNA.0b013e3182346e4b.

7. Lobão WM, Menezes IG. Construção e validação de conteúdo da escala de predisposição à ocorrência de eventos adversos. Rev. Latino-Am. Enfermagem. [Internet] 2012;20(4) [acesso em 13 fev 2016]. Disponível: http:// dx.doi.org/10.1590/S0104-11692012000400021.

8. Cassano-Piché A, Fan M, Sabovitch S, Masino C, Easty AC, Health Technology Safety Research Team; et al. Multiple intravenous infusions - Phase 1b: practice and training scan. Ont Health Technol Assess Ser.[Internet] 2012;12(16)[acesso em 12 fev 2016]. Disponível: https://www.ncbi.nlm.nih.gov/pmc/articles/PMC3377572/.

9. Lemoine JB, Hurst HM. Using smart pumps to reduce medication errors in the NICU. Nurs WomHealth. [Internet] 2012;16(2)[acesso em 14 fev 2016]. Disponível: http://dx.doi.org/10.1111/j.1751-486X.2012.01721.x.

10. Reis CT, Laguardia J, Martins M. Adaptação transcultural da versão brasileira do Hospital Survey on Patient Safety Culture: etapa inicial. Cad. Saúde Pública. [Internet] 2012;28(11) [acesso em 13 fev 2016]. Disponível: http:// www.scielosp.org/pdf/csp/v28n11/19.pdf.

11. Kuroda T, Noma H, Nalto C, Tada M, Yamanaka H, Takemura T, et al. Prototyping sensor network system for automatic vital signs collection. Methods Inf Med. [Internet] 2013;52(3) [acesso em 10 fev 2016]. Disponível: http://dx.doi.org/10.3414/ME12-01-0096.

12. Pires MPO, Pedreira MLG, Peterlini MAS.Cirurgia segura em pediatria: elaboração e validação de checklist de intervenções pré-operatórias.Rev. Latino-Am. Enfermagem. [Internet] 2013;21(5)[acesso em 14 fev 2016]. Disponível: http://dx.doi.org/10.1590/S0104-11692013000500010.

14. Siqueira LDC, Caliri MHL, Kalisch B, Dantas RAS. Adaptação cultural e análise da consistência interna do instrumento MISSCARE para uso no Brasil. Rev Latino-Am. Enfermagem. [Internet] 2013;21(2) [acesso em 10 fev 2016]. Disponível: http://dx.doi.org/10.1590/S0104-11692013000200019.

15. Oliveira RM, Leitão IMTA, Figueiredo SV, Sampaio RL, Gondim MM. Estratégias para promover a segurança do paciente: da identificação dos riscos às praticas baseadas em evidências. Esc. Anna Nery. [Internet] 2014;18(1) [acesso em 10 fev 2016]. Disponível: http://dx.doi.org/10.5935/1414-8145.20140018.

16. Uneke, CJ, Ndukwe CD, Oyibo PG, Nwakpu KO, Nnabu RC, Prasopa-Plaizier N. Promotion of hand hygiene strengthening initiative in a Nigerian teaching hospital: implication for improved patient safety in low-income health facilities. Braz J Infect Dis. [Internet] 2014;18(1)[acesso em 10 fev 2016]. Disponível: http://dx.doi. org/10.1016/j.bjid.2013.04.006.

17. Simonsen BO, Daehlin GK, Johansson I, Farup PG. Improvement of drug dose calculations by classroom teaching or e-learning: a randomised controlled trial in nurses. BMJ Open. [Internet] 2014;4(10)[acesso em 10 fev 2016]. Disponível: http://dx.doi.org/10.1136/bmjopen-2014-006025.

18. Luzia MF, Almeida MA, Lucena AF. Mapeamento de cuidados de enfermagem para pacientes com risco de quedas na Nursing Interventions Classification. Rev.esc. enferm. USP. [Internet] 2014;48(4)[acesso em 10 fev 2016]. Disponível: http://dx.doi.org/10.1590/S0080-623420140000400009.

19. de Souza S, Rocha PK, Cabral PFA, Kusahara DM. Utilização de estratégias de segurança na identificação da criança para administração de medicamentos. Acta paul. enferm. [Internet] 2014;27(1) [acesso em 10 fev 2016]. Disponível: http://dx.doi.org/10.1590/1982-0194201400003. 
20. Barra DCC, Dal Sasso GTM, Baccin CRA. Sistemas de alerta em um processo de enfermagem informatizado para Unidades de Terapia Intensiva. Rev. esc. enferm. USP.[Internet] 2014;48(1)[acesso em 13 fev 2016]. Disponível: http://dx.doi.org/10.1590/S0080-623420140000100016.

21. Barbosa TP, de Oliveira GAA, Lopes MNA, Poletti NAA, Beccaria LM. Práticas assistenciais para segurança do paciente em unidade de terapia intensiva. Actapaul. enferm. [Internet] 2014;27(3)[acesso em 10 fev 2016]. Disponível: http://dx.doi.org/10.1590/1982-0194201400041.

22. Donaldson N, Aydin C, Fridman M, Foley M. Improving medication administration safety: using naïve observation to assess practice and guide improvements in process and outcomes. J HealthcQuality. [Internet] 2014;36(6) [acesso em 10 fev 2016]. Disponível: http://dx.doi.org/10.1111/jhq.12090.

23. Alsweed F, Alshaikh A, Ahmed A, Yunus F, Househ M. Impact of computerised provider order entry system on nursing workflow, patient safety, and medication errors: perspectives from the front line. Int J Electron Healthc. [Internet] 2014;7(4) [acesso em 10 fev 2016]. Disponível: http://dx.doi.org/10.1504/IJEH.2014.064328.

24. da Silva R, Amante LN. Checklist para o transporte intra-hospitalar de pacientes internados na unidade de terapia intensiva. Texto Contexto Enferm. [Internet] 2015;24(2) [acesso em 15 fev 2016]. Disponível: http://dx.doi. org/10.1590/0104-07072015001772014.

25. de Magalhães AMM, de Moura GMSS, Pasin SS, Funcke LB, Pardal BM, Kreling A. Processos de medicação, carga de trabalho e a segurança do paciente em unidades de internação. Rev. esc. enferm. USP. [Internet] 2015;49(n.esp)[acesso em 10 fev 2016]. Disponível: http://dx.doi.org/10.1590/S0080-623420150000700007.

26. Howick J, Chalmers I, Glasziou P, Greenhalgh T, Heneghan C, Liberati A, et al. The Oxford Levels of Evidence 2. Oxford Centre for Evidence-Based Medicine.[Internet] 2011 [acessoem 13 fev 2016]. Disponível: http://www. cebm.net/ocebm-levels-of-evidence. 